\title{
Efficacy of docetaxel plus ramucirumab as palliative second-line therapy following first-line chemotherapy plus immune-checkpoint-inhibitor combination treatment in patients with non-small cell lung cancer (NSCLC) UICC stage IV
}

\author{
Wolfgang M. Brueckl ${ }^{1,2}$, Martin Reck ${ }^{3}$, Achim Rittmeyer ${ }^{4}$, Jens Kollmeier ${ }^{5}$, Claas Wesseler ${ }^{6}$, \\ Gunther H. Wiest ${ }^{6}$, Petros Christopoulos ${ }^{7}$, Albrecht Stenzinger ${ }^{8,9}$, Amanda Tufman ${ }^{10}$, Petra Hoffknecht ${ }^{11}$, \\ Bernhard Ulm $^{12}$, Fabian Reich ${ }^{1,2}$, Joachim H. Ficker ${ }^{1,2}$, Eckart Laack ${ }^{13}$ \\ ${ }^{1}$ Department of Respiratory Medicine, Allergology and Sleep Medicine/Nuernberg Lung Cancer Center, Nuernberg General Hospital, Nuremberg, \\ Germany; ${ }^{2}$ Paracelsus Medical University Nuremberg, Nuremberg, Germany; ${ }^{3}$ Department of Thoracic Oncology, Airway Research Center North, \\ German Center for Lung Research, Lung Clinic, Grosshansdorf, Germany; ${ }^{4}$ Lungenfachklinik Immenhausen, Ambulanz für pneumologische \\ Onkologie, Kassel, Germany; ${ }^{5}$ Helios Klinikum Emil von Behring, Lungenklinik Heckeshorn, Berlin, Germany; ${ }^{6}$ Asklepios Tumorzentrum \\ Hamburg, Klinikum Harburg, Hamburg, Germany; ${ }^{7}$ Thoraxklinik-Heidelberg gGmbH, Heidelberg, Germany; ${ }^{8}$ Institute of Pathology, University \\ Hospital Heidelberg, Heidelberg, Germany; ${ }^{9}$ German Cancer Consortium (DKTK), Partner Site Heidelberg, and German Cancer Research \\ Center (DKFZ), Heidelberg, Germany; ${ }^{10}$ Medizinische Klinik V, Pneumologie, Klinikum der Universität München, Member of the German Center \\ for Lung Research, CPC-M, Munich, Germany; ${ }^{11}$ Lungenzentrum Osnabrueck, Klinik für Thoraxonkologie, Franziskus-Hospital Harderberg, \\ Georgsmarienhütte, Germany; ${ }^{12}$ Unabhängige statistische Beratung Bernhard Ulm, Munich, Germany; ${ }^{13}$ Hämato-Onkologie Hamburg, Hamburg, \\ Germany \\ Contributions: (I) Conception and design: WM Brueckl, E Laack; (II) Administrative support: E Laack, JH Ficker; (III) Provision of study materials \\ or patients: M Reck, A Rittmeyer, J Kollmeier, C Wesseler, GH Wiest, P Christopoulos, A Tufman, P Hoffknecht, F Reich; (IV) Collection and \\ assembly of data: WM Brueckl; (V) Data analysis and interpretation: B Ulm, A Stenzinger, WM Brueckl; (VI) Manuscript writing: All authors; \\ (VII) Final approval of manuscript: All authors. \\ Correspondence to: Wolfgang M. Brueckl, MD. Department of Respiratory Medicine, Allergology and Sleep Medicine, Nuernberg General Hospital; \\ Paracelsus Medical University, Ernst-Nathan-Str. 1, 90419 Nuremberg, Germany. Email: wolfgang.brueckl@klinikum-nuernberg.de.
}

Background: Chemotherapy plus immune-checkpoint inhibitor (CTx+ICI) therapy has become the preferred 1st line treatment in patients with metastatic NSCLC without oncogenic driven mutations. However, the optimal subsequent 2 nd line treatment is not defined and several alternatives exist. The purpose of this analysis was to evaluate the efficacy of 2 nd line docetaxel plus ramucirumab (D+R) initiated after failure of 1 st line CTx+ICI.

Methods: Retrospective data were collected during routine care from German thoracic oncology centers. Only patients who had received at least one course of 2 nd line $\mathrm{D}+\mathrm{R}$ were included. ORR, PFS, OS and numbers of courses of $\mathrm{D}+\mathrm{R}$ were investigated with $\mathrm{PFS}$ after initiation of $\mathrm{D}+\mathrm{R}$ being the primary endpoint.

Results: Seventy-seven patients met the inclusion criteria. 2 nd line treatment with $D+R$ achieved an ORR and DCR of $32.5 \%$ and $62.4 \%$, respectively. Median PFS for 2 nd line therapy was 3.9 months with a DOR of 6.4 months. Median OS of 15.5 and 7.5 months were observed from the start of 1 st line therapy and 2 nd line treatment, respectively. No unexpected toxicities occurred. Presence of KRAS mutations was associated with significantly worse median PFS to $\mathrm{D}+\mathrm{R}$ ( 2.8 vs. 4.5 months in wild-type cases; $\mathrm{P}=0.021)$ and was an independent predictor of inferior PFS in multivariate analysis.

Conclusions: D+R is an effective and safe 2nd line treatment after failure of 1 st line CTx+ICI irrespective of NSCLC histology. However, patients with a KRAS mutation did not benefit from D+R in terms of PFS

$\wedge$ ORCID: 0000-0002-7039-0791. 
and will require further investigations.

Keywords: Lung cancer; palliative treatment; angiogenesis inhibitor; ramucirumab; immune-checkpoint inhibitor (ICI)

Submitted Mar 11, 2021. Accepted for publication May 25, 2021.

doi: $10.21037 /$ tlcr-21-197

View this article at: https://dx.doi.org/10.21037/tlcr-21-197

\section{Introduction}

In recent years, ICI directed against PD-1 or PD-L1 have expanded the treatment options for advanced NSCLC. Initially, ICI were approved for palliative $2 \mathrm{nd}$ and $3 \mathrm{rd}$ line treatment of metastatic NSCLC in patients without druggable driver mutations (1-6). Up to $16 \%$ of NSCLC patients receiving the $\mathrm{PD}-1$ inhibitor nivolumab as palliative 2nd line treatment survive for more than five years (7). ICI quickly became established in the 1st line setting. The PD-1 ICI pembrolizumab is approved in Europe as a 1st line monotherapy for patients with an immunohistochemically based tumor proportion score (TPS) for PD-L1 $\geq 50 \%$ or in combination with a platinum based combination chemotherapy regardless of the TPS score (8-10). Currently approved 1st line therapies also include the PD-L1 ICI atezolizumab either in combination with chemotherapy or even as a four-drug regimen in combination with the VEGF antibody bevacizumab $(11,12)$. In addition, the PD-1 antibody nivolumab is approved in combination with the CTLA-4 ICI ipilimumab and platinum-based combination therapy (13). These ICI combination strategies have resulted in a median PFS rates of 7 to 9 months and median OS rates of 16 to 22 months. Most patients will sooner or later experience progression of their tumor disease and require 2 nd line treatment. However, there is a lack of knowledge about the efficacy of drugs being considered for 2nd line therapy following CTx+ICI, since the current options were all tested and approved before the widespread use of immunotherapy. It is currently unclear to what extent the results of former trials can be extrapolated to inform the choice of 2 nd line therapy after immunotherapy (14).

So far, no studies have prospectively compared different therapy strategies in 2 nd line. Thus, real-world data from high-volume centers with well-defined treatment sequences could help physicians to make treatment decisions in individual NSCLC patients. Recently we could show excellent efficacy of 3 rd line docetaxel plus ramucirumab $(\mathrm{D}+\mathrm{R})$ combination therapy after failure of both 1 st line chemotherapy and 2nd line ICI monotherapy (15). In this retrospective analysis we now report on $\mathrm{D}+\mathrm{R}$ in the palliative 2 nd line treatment after progression on the current standard 1st line treatment comprising of platinum based combination chemotherapy plus ICI $(\mathrm{CTx}+\mathrm{ICI})$. We present the following article in accordance with the STROBE reporting checklist (available at https://dx.doi. org/10.21037/tlcr-21-197).

\section{Methods}

\section{Design and participating centers}

This retrospective analysis studies the clinical effects of a palliative 2 nd line treatment with $\mathrm{D}+\mathrm{R}$ in patients with metastatic NSCLC directly after progression on a 1st line combination therapy with platinum based chemotherapy and an anti-PD-1 or PD-L1 ICI agent $(\mathrm{CT}+\mathrm{ICI})$. Patients had a histologically or cytologically proven NSCLC UICC stage IV according to the eight edition of the UICC TNM classification. Patients with ICI monotherapy as 1st line therapy were excluded. Patients with a history of adjuvant chemotherapy before 1 st line palliative therapy could be included, if there was an interval of more than 12 months between the adjuvant treatment and the first systemic treatment for stage IV disease. Patients with a history of palliative radiation therapy could also be enrolled into the study. NSCLC with a non-squamous histology were tested for EGFR mutations and ALK translocations and in case of a positive result excluded.

Data were collected from 9 high-volume German centers with specialization in thoracic oncology and strong experience in chemo- and immune-oncology therapy. The centers included three university hospitals, three community-based hospitals, two private hospitals and one outpatient clinic. Patients were treated with $\mathrm{CTx}+\mathrm{ICI}$ between February 2016 and May 2020 and started 2nd line $\mathrm{D}+\mathrm{R}$ no later than August, 1st 2020. This allowed for a follow-up of at least five months prior to the data cut- 
off of December 31, 2020. Data were recorded by each center in a standardized manner with the following data being collected for each patient: age, sex, smoking habits, tumor stage, histology, PD-L1 expression determined by immunohistochemistry, mutational status, height and weight at diagnosis, date of NSCLC diagnosis, survival status at time point of documentation (alive or deceased) and date of last contact/death. For each treatment line the following details of therapy were documented: date of start, number of treatment cycles, cycles for combination and monotherapy, best response, date of progression and reason for treatment stop. In addition, for 2 nd line therapy the following data were documented: weight at start of therapy and side effects [according to common toxicity criteria (CTC) grades 3 and 4].

Data were anonymized before being sent to the organizing center. Inclusion and exclusion criteria were verified centrally, and data were checked for completeness and plausibility. This study was conducted in accordance with the Declaration of Helsinki (as revised in 2013). This study was approved by the Institutional Review Board of Paracelsus Medical University Nuremberg (IRB-2019-014). Due to the retrospective nature of the study and the strict anonymization of individual data, written informed consent was not required.

\section{Measurement of immunobistochemical and molecular factors}

PD-L1 expression was assessed with the following antibodies SP263 (6 centers), ZR3 (1 center), 22C3 (1 center) and QR001 (1 center). Tumor proportional scores (TPS) were classified into three groups $(<1 \%, 1-49 \%$ and $\geq 50 \%$ ). The genetic make-up of tumors was analyzed using tissue-based targeted next-generation-sequencing (NGS).

\section{Evaluation of tumor response and efficacy of treatment}

The primary endpoint was efficacy of the $\mathrm{D}+\mathrm{R} 2 \mathrm{nd}$ line therapy in terms of progression-free survival (PFS). Secondary endpoints were duration of response (DOR) in patients responding to $\mathrm{D}+\mathrm{R}$ as well as OS from start of 2 nd line therapy. In addition, response to 1 st and 2 nd line treatment, time interval between both lines of treatment and the relationship between tumor response to $\mathrm{CTx}+\mathrm{ICI}$ and $\mathrm{D}+\mathrm{R}$ were evaluated.

Tumor responses were assessed by each center by chest computer tomography (CT) and abdominal ultrasound or other clinically appropriate abdominal imaging at least every 3 months or upon clinical deterioration. Tumor responses were evaluated according to the RECIST (Response Evaluation Criteria in Solid Tumors) version 1.1. (16). Complete response (CR) was defined as disappearance of all target and non-target lesions. Partial response (PR) was defined as $\geq 30 \%$ reduction in size in target lesions or disappearance of $\geq 1$ non-target lesions. Stable disease (SD) was defined as $<30 \%$ decrease or $<20 \%$ increase in size of target lesions or the persistence of $\geq 1$ non-target lesions. Progressive disease (PD) was defined as $\geq 20 \%$ increase in size or the appearance of new non-target lesions and/ or progression of existing non-target lesions. The ORR was defined as the best response recorded from the start of treatment until disease progression or recurrence, confirmed by repeat assessments performed no less than four weeks after the first time criteria for response had been reached. Disease control rate was defined as CR plus PR plus SD.

Overall survival was recorded from the first day of 1 st line palliative treatment with $\mathrm{CT} x+\mathrm{ICI}$ and from the first day of 2 nd line treatment with $\mathrm{D}+\mathrm{R}$ to the date of death or last follow-up. PFS was defined for 2 nd line therapy as the interval from the first day of $2 \mathrm{nd}$ line $\mathrm{D}+\mathrm{R}$ to the first sign of disease progression or death whichever occurred first. For 1st line therapy, time on treatment was defined as the interval starting from the first day on drug until the date when the progression was documented.

\section{Statistical analysis}

Descriptive data were presented as mean and $95 \%$ confidence interval, categorical variables were presented using numbers and frequencies. A tornado diagram was implemented to visualize the relative importance of cycle length in 1st and 2nd line treatment. To analyze PFS and OS data were presented as median and $95 \%$ confidence interval (CI), and times to events were determined using the Kaplan-Meier method and compared with the log-rank test. Cox proportional hazards models were used to evaluate the influence of different patient factors. Statistical results were calculated using SPSS (version 23). A P value $<0.05$ was considered statistically significant.

\section{Results}

\section{Patient population}

After excluding 10 patients (5 patients with ICI 
Table 1 Patients' demographics

\begin{tabular}{|c|c|c|}
\hline Parameters & $\mathrm{N}$ & $(\%)$ \\
\hline \multicolumn{3}{|l|}{ Age } \\
\hline \multicolumn{3}{|c|}{ Median age 63 years (range, $41-83$ ) } \\
\hline$<65$ years & 41 & $(53.2)$ \\
\hline$\geq 65$ years & 36 & $(46.8)$ \\
\hline \multicolumn{3}{|l|}{ Gender } \\
\hline Male & 53 & $(68.8)$ \\
\hline Female & 24 & $(31.2)$ \\
\hline \multicolumn{3}{|l|}{ ECOG PS } \\
\hline 0 & 28 & $(38.4)$ \\
\hline 1 & 40 & $(54.8)$ \\
\hline 2 & 5 & $(6.8)$ \\
\hline n.r. & 4 & \\
\hline \multicolumn{3}{|c|}{ Stage at start of 1st line chemotherapy } \\
\hline IVa & 20 & $(26.0)$ \\
\hline $\mathrm{IVb}$ & 57 & $(74.0)$ \\
\hline \multicolumn{3}{|l|}{ Histology } \\
\hline Adenocarcinoma & 55 & $(71.4)$ \\
\hline Squamous cell carcinoma & 16 & $(20.8)$ \\
\hline NOS or other type & 6 & $(7.8)$ \\
\hline \multicolumn{3}{|l|}{ PDL-1 expression status } \\
\hline Negative & 33 & $(47.1)$ \\
\hline $1-49 \%$ & 30 & $(42.9)$ \\
\hline$\geq 50 \%$ & 7 & $(10.0)$ \\
\hline n.r. & 7 & \\
\hline \multicolumn{3}{|l|}{ Kras mutational status } \\
\hline Wild-type & 31 & $(64.6)$ \\
\hline Mutation & 17 & $(35.4)$ \\
\hline n.r. & 29 & \\
\hline \multicolumn{3}{|l|}{ BMI at start of 2 nd line } \\
\hline$<25$ & 44 & $(57.1)$ \\
\hline$\geq 25$ & 33 & $(42.9)$ \\
\hline \multicolumn{3}{|l|}{ Palliative radiation therapy } \\
\hline No radiation & 37 & $(48.1)$ \\
\hline
\end{tabular}

Table 1 (continued)
Table 1 (continued)

\begin{tabular}{lcl}
\hline Parameters & $\mathrm{N}$ & $(\%)$ \\
\hline One site & 10 & $(13.0)$ \\
Brain & 7 & $(9.1)$ \\
Bone & 9 & $(11.7)$ \\
Lung/mediastinum & 4 & $(5.2)$ \\
Multiple sites & 10 & $(13.0)$ \\
n.r. &
\end{tabular}

n.r., not reported; BMI, body mass index.

monotherapy in 1st line; 3 patients with $\mathrm{D}+\mathrm{R}$ in the $3 \mathrm{rd}$ line, 2 patients with insufficient follow-up data), 77 patients from 9 centers met the inclusion criteria. The baseline demographic data and tumor characteristics are listed in Table 1 . The median age was 63 years (range, 41-83), and $68.8 \%$ of the patients were male. More than half of the patients had an ECOG-PS (Eastern Cooperative Oncology Group performance status) of 1 (54.8\%). PD-L1 data were available for 70 patients (91\%); of those $47.1 \%$, $42.9 \%$ and $10 \%$ exhibited a tumor proportion score (TPS) of $0 \%$, $1-49 \%$ and $\geq 50 \%$, respectively. The KRAS mutational status was available from 48 tumors $(68.6 \%)$; of those a KRAS mutation was detected in 17 (35.4\%) of the cases. A G12C mutation was identified in 5 cases (29.4\%). KRAS G12V, G12D, G12A, G12S and a codon 13 mutation were observed in 5, 3, 2,1 and 1 cases, respectively. All patients had received a platinum based combination $\mathrm{CTx}+\mathrm{ICI}$ as palliative 1 st line therapy with carboplatin being the backbone in $83.1 \%$. ICI included pembrolizumab in $80.5 \%$, atezolizumab in $13 \%$ and durvalumab (due to a clinical study) in $6.5 \%$. In 20 patients a $3 \mathrm{rd}$ line therapy was initiated following D+R. 3rd line therapy consisted of combination CTx, CTx monotherapy, ICI monotherapy and a tyrosine kinase inhibitor (TKI) were given in $8,3,6$ and 3 cases, respectively. Details of agents and combinations are reported in Table 2.

\section{Efficacy and safety of $D+R$}

The mean number of 2 nd line combination treatment cycles was 6.0 (95\% CI, 5.1-7.0) with a mean number of 2.1 cycles given as a ramucirumab mono therapy. Fifteen patients $(19.5 \%)$ were still on treatment with $D+R$ at 
Table 2 Drugs and drug combinations used in different lines of treatment

\begin{tabular}{|c|c|c|c|c|c|}
\hline \multicolumn{2}{|l|}{ 1st line } & \multicolumn{2}{|l|}{ 2nd line } & \multicolumn{2}{|l|}{ 3rd line therapy } \\
\hline Platinum + pemetrexed + pembrolizumab & $50(64.9)$ & & & & \\
\hline $\begin{array}{l}\text { Platinum + paclitaxel/nab-paclitaxel + } \\
\text { pembrolizumab }\end{array}$ & $9(11.7)$ & Docetaxel + ramucirumab & 77 [100] & Chemotherapy mono & $8[40]$ \\
\hline $\begin{array}{l}\text { Platinum + gemcitabine/vinorelbine + } \\
\text { durvalumab + tremelimumab }\end{array}$ & $3(3.9)$ & & & $\mathrm{ICl}$ & $6[30]$ \\
\hline $\begin{array}{l}\text { Platinum + pemetrexed + durvalumab } \\
\text { +tremelimumab }\end{array}$ & $2(2.6)$ & & & TKI & $3[15]$ \\
\hline $\begin{array}{l}\text { Platinum + gemcitabine/vinorelbine + } \\
\text { pembrolizumab }\end{array}$ & $1(1.3)$ & & & & \\
\hline
\end{tabular}

$\mathrm{ICI}$, immune-checkpoint-inhibitor; TKI, tyrosine kinase inhibitor.

data cut-off. Overall, this treatment led to an ORR of $32.5 \%$ and a DCR of $62.4 \%$. The median PFS for 2 nd line therapy was 3.9 months (95\% CI, 3.1-4.6) with a DOR of 6.4 months (95\% CI, 5.4-7.4) (Table 3 and Figure $1 A$ ). There was no significant difference in PFS between patients with squamous or non-squamous tumor histology receiving $\mathrm{D}+\mathrm{R}$ (Table 3, Figure 1B). The median OS from first dose of 2 nd line therapy was 7.5 months (95\% CI, 5.1-10.0). A median OS of 15.5 months (95\% CI, 12.2-18.9) was observed from the start of 1 st-line palliative treatment (Figure 2). Cox regression analyses were carried out to analyze potential prognostic effects on PFS including gender, age, stage, BMI, histology, response to 1 st line treatment, PD-L1 TPS and KRAS mutational status. PD-L1 TPS and KRAS mutational status were prognostic in the univariate analysis. Only KRAS wild-type status turned out to be of independent positive prognostic value (Figure $3 A, B$, Table 4). There was no difference in terms of efficacy between patients with KRAS G12C and other KRAS mutations (Figure S1).

During $\mathrm{D}+\mathrm{R}$ treatment no unexpected toxicity was documented. Neutropenia was the most frequent side effect with CTC grades 3 and 4 documented in 7 and 5 patients, respectively. Febrile neutropenia was reported in 3 patients, 2 of them suffering from CTC grade
4 leading to discontinuation of the $\mathrm{D}+\mathrm{R}$ treatment. Prophylactic G-CSF was not routinely administered in any of the centers. However, in patients with grade 3 or 4 neutropenia prophylactic G-CSF was given for subsequent courses to prevent further hematologic adverse events. Alternatively, in some cases docetaxel was discontinued and ramucirumab was given as a mono-therapy. Further commonly documented grade 3-4 CTC toxicities included fatigue, dysparonychia, mucositis, stomatitis, and ileus in 5, 4, 3, 1 and 1 cases, respectively. No pulmonary toxicities, such as interstitial pneumonitis, or treatment-related deaths were observed. The adverse events associated with 2 nd line therapy are listed in Table 5.

\section{Outcome of 1st line treatment}

A response to 1 st line therapy was observed in $50.7 \%$ of patients. The mean time on 1st line treatment was 5.8 months (95\% CI, 5.0-6.6). After an average of 4 cycles of platinumbased CTX+ICI, 37.8\% received a maintenance therapy with $\mathrm{CT}+\mathrm{ICI}$ and $41 \%$ received ICI monotherapy (Table 3). There were no significant differences in OS between the different platinum agents. Regarding PD-L1 TPS and duration of treatment with ICI there was a trend toward longer duration of therapy in patients with a high expression $(\geq 50 \%)$. The 
Table 3 Efficacy data in different lines of treatment

\begin{tabular}{|c|c|c|c|}
\hline Parameters & 1st line $\mathrm{CT} x+\mathrm{ICl}$ & 2nd line $R+D$ & 3rd line \\
\hline Number of cycles; mean $(95 \% \mathrm{Cl})$ & $9.0(7.4-10.6)$ & $6.0(5.1-7.0)$ & - \\
\hline \multicolumn{4}{|l|}{ Of those } \\
\hline Maintenance $\mathrm{CT} x+\mathrm{ICl}$ & $2.0(1.2-2.8)$ & $2.1(1.3-3.0)$ & \\
\hline Ramucirumab mono & - & & \\
\hline \multicolumn{4}{|l|}{ ORR; N (\%) } \\
\hline $\mathrm{CR}$ & $3(3.9)$ & & \\
\hline PR & $36(46.8)$ & $25(32.5)$ & $2(2.6)$ \\
\hline \multirow[t]{2}{*}{ n.r. } & - & $6(7.8)$ & $6(7.8)$ \\
\hline & & & $\begin{array}{l}\text { 2nd line ongoing } 15(19.5) \text {; } \\
\text { no further therapy } 42(54.5)\end{array}$ \\
\hline DOR; median (95\% Cl) & $8.7(6.3-11.0)$ & $6.4(5.4-7.4)$ & - \\
\hline PFS; median (95\% Cl) (months) & $5.8(5.0-6.6)$ & $3.9(3.1-4.6)$ & - \\
\hline \multicolumn{4}{|l|}{ PFS according to subgroups } \\
\hline \multicolumn{4}{|l|}{ Histology } \\
\hline Adenocarcinoma & $6.0(5.0-7.0)$ & $3.9(3.0-4.8)$ & - \\
\hline \multicolumn{4}{|l|}{ KRAS } \\
\hline Wild-type & $6.0(4.8-7.2)$ & $4.5(2.6-6.4)$ & - \\
\hline Mutated & $4.8(3.2-6.3)$ & $2.8(1.7-3.9)$ & - \\
\hline OS from line of thx; median $(95 \% \mathrm{Cl})$ (months) & $15.5(12.2-18.9)$ & $7.5(5.1-10.0)$ & $3.4(2.6-4.2)$ \\
\hline
\end{tabular}

TPS, tumor proportional score on immunohistochemistry; DOR, duration of response.

median time interval from end of 1st line to start of 2 nd line treatment was 23 days (95\% CI, 19.6-26.4). There was no statistically significant effect of this interval on the efficacy of 2 nd line D+R. Radiation therapy had no significant effect on 1 st or 2 nd line response or outcome. However, BMI appeared to be of prognostic value in terms of OS. From the start of 1st line median OS was 21.4 months in patients with initial $\mathrm{BMI} \geq 25$ and 13.8 months in patients with $\mathrm{BMI}<25$
$(\mathrm{P}=0.018)$. BMI did not affect clinical response or PFS in either treatment line.

\section{Subgroup analysis of patients treated with platinum + pemetrexed + pembrolizumab in the 1st line}

Nearly two-thirds of the patients $(\mathrm{N}=50 ; 64.9 \%)$ had been treated with $\mathrm{CTx}+\mathrm{ICI}$ consisting of platinum + pemetrexed 
A

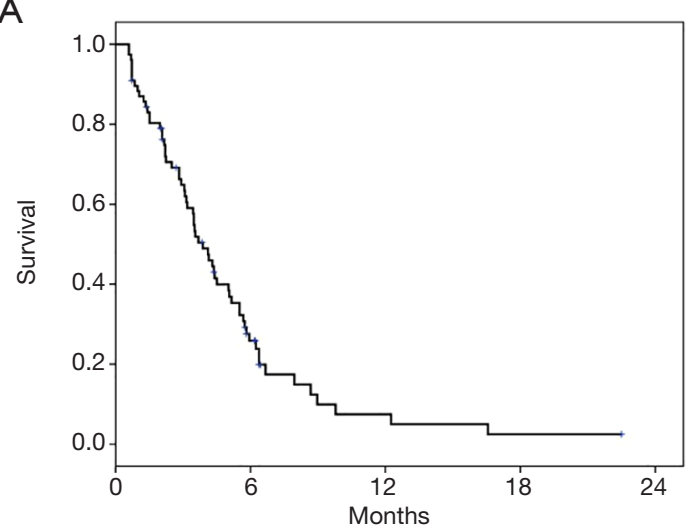

B

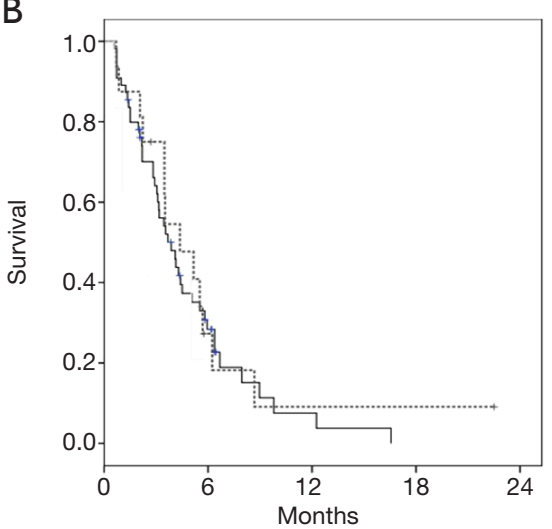

$P=0.350$

ᄃ Adenocarcinoma

Squamous cell carcinoma not otherwise determined

+ Adenocarcinoma-censored

+ Squamous cell carcinomacensored

not otherwise determined-censored

Figure 1 Kaplan-Meier curve for PFS. (A) PFS Kaplan Meier curves of the whole collective, (B) PFS due to different histologies, ADC, adeno carcinoma; SCC, squamous cell carcinoma.
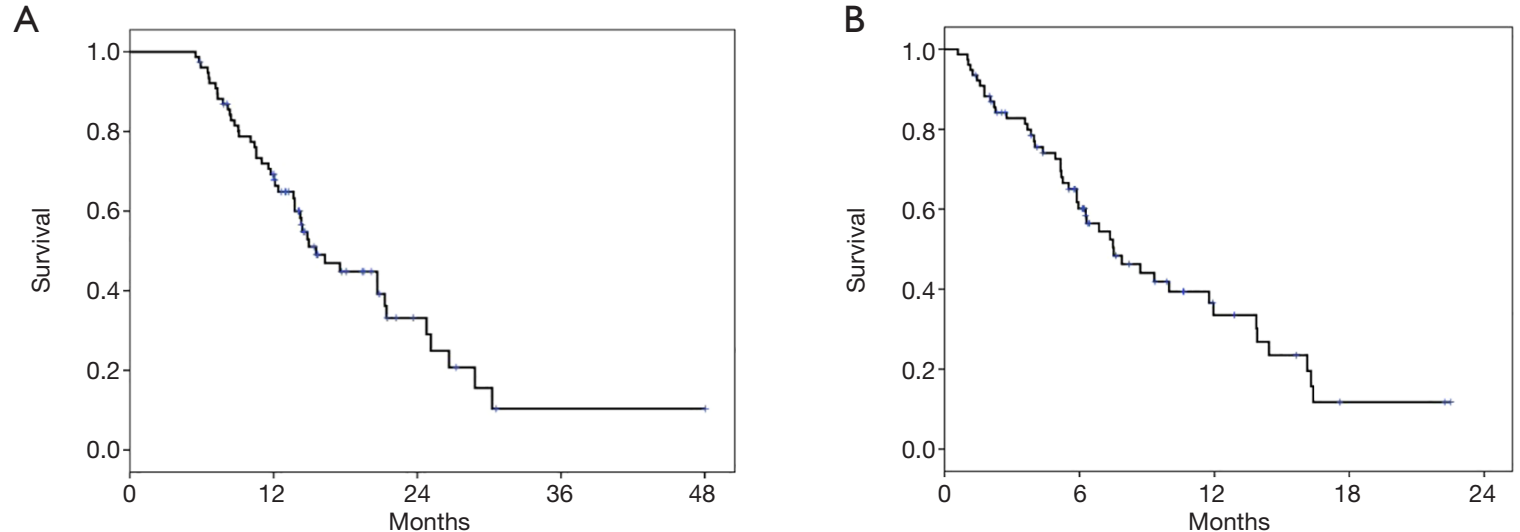

Figure 2 Kaplan-Meier curves for OS due to start of (A) 1st line and (B) 2nd line treatment, respectively.

A

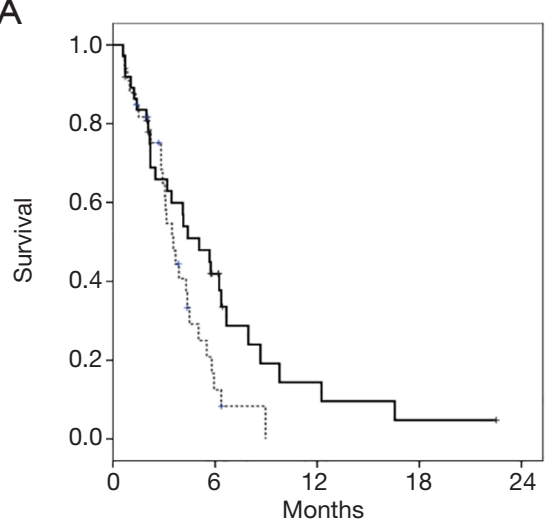

B

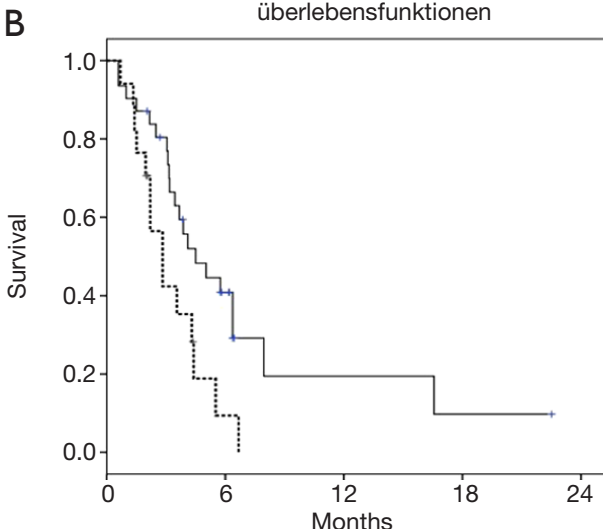

$\mathrm{P}=0.018$

$\neg$ KRAS wildtype KRAS mutation + KRAS wildtype-censored + KRAS mutation-censored

Figure 3 Kaplan-Meier curves for molecular subgroups. PFS. (A) PFS due to PD-L1 TPS scores; (B) PFS due to KRAS mutational status. mut, KRAS mutated; wt, KRAS wild-type. 
Table 4 Univariate and multivariate analysis of different parameters of the whole cohort regarding PFS due to D+R treatment

\begin{tabular}{|c|c|c|c|c|c|c|c|c|c|}
\hline Parameters & $\mathrm{N}$ & mPFS & $95 \% \mathrm{Cl}$ & \multicolumn{3}{|c|}{ Univariate analysis } & \multicolumn{3}{|c|}{ Multivariate analysis } \\
\hline \multicolumn{10}{|l|}{ Gender } \\
\hline Male & 53 & 3.7 & $2.6-4.7$ & & & & & & \\
\hline Female & 24 & 5.0 & $1.7-8.3$ & 0.682 & $0.392-1.186$ & 0.175 & - & & \\
\hline$<65$ & 41 & 4.5 & $2.8-6.2$ & & & & & & \\
\hline$\geq 65$ & 36 & 3.5 & $3.0-4.0$ & 1.545 & $0.910-2.598$ & 0.101 & - & & \\
\hline \multicolumn{10}{|l|}{ Stage } \\
\hline IV A & 20 & 5.0 & $2.6-7.5$ & & & & & & \\
\hline$<25$ & 44 & 3.7 & $1.9-5.6$ & & & & & & \\
\hline$\geq 25$ & 33 & 4.3 & $2.1-6.5$ & 0.702 & $0.417-1.179$ & 0.181 & - & & \\
\hline \multicolumn{10}{|l|}{ Histology } \\
\hline$A C$ & 55 & 3.9 & $2.8-4.9$ & & & & & & \\
\hline SCC & 16 & 4.4 & $2.3-6.4$ & & & & & & \\
\hline NOS & 6 & 2.5 & $0-5.5$ & 1.064 & $0.850-1.318$ & 0.568 & - & & \\
\hline \multicolumn{10}{|c|}{ Response 1st line } \\
\hline Response & 39 & 4.5 & $2.8-4.1$ & & & & & & \\
\hline \multicolumn{10}{|l|}{ KRAS } \\
\hline Wild-type & 31 & 4.5 & $2.6-6.4$ & & & & & & \\
\hline Mutation & 17 & 2.8 & $1.7-3.9$ & 2.293 & $1.130-4.652$ & 0.021 & 2.229 & $1.077-4.605$ & 0.030 \\
\hline
\end{tabular}

+ pembrolizumab in 1 st line therapy. All of these patients had a non-squamous tumor and in $84 \%$ the mutational status had been established. Thus, these patients represent a large, homogeneous, and well-characterized subgroup and were therefore analyzed in more detail in a subsequent post-hoc analysis. A tornado-plot representing the number of cycles in 1st and 2nd line treatment is given in Figure 4.

The platinum backbone was carboplatin and cisplatin in $78 \%$ and $22 \%$, respectively, with no differences in duration of treatment in 1st line or 2 nd line. PD-L1 TPS was $0 \%, 1-49 \%$ and $\geq 50 \%$ in $48 \%, 46 \%$ and $6 \%$ of the tumors, respectively, with no differences in outcomes. 2nd line treatment with $\mathrm{D}+\mathrm{R}$ resulted in an ORR of $34 \%$ and a DCR of $68 \%$, with a DOR of 6.4 months (95\% CI, 5.6-7.2 months). The mPFS for $\mathrm{D}+\mathrm{R}$ was 3.9 months (2.7-5.1 months) and the mOS from the start of 1st and 2nd line was 16.3 and 8.7 months, respectively. The KRAS status of the tumor was reported for 42 patients and a KRAS mutation was detected in 16 cases (38.1\%) (Figure 4). KRAS mutations were significantly associated with poor median PFS for $\mathrm{D}+\mathrm{R}$ in contrast to the 
wild type status ( $2.8 v$ s. 5.0 months; $\mathrm{P}=0.023$ ). As described for the entire cohort, there was no difference between KRAS G12C and other KRAS mutations when comparing PFS in this subgroup. The duration of treatment in 1st line or

Table 5 Hematological and non-hematological side effects of $\mathrm{D}+\mathrm{R}$ therapy

\begin{tabular}{lc}
\hline Adverse events & CTC grade $\geq 3^{*}, \mathrm{~N}(\%)$ \\
\hline Neutropenia & $12(15.6)$ \\
Febrile neutropenia & $3(3.9)$ \\
Fatigue & $5(6.5)$ \\
Dysparonychia & $4(5.2)$ \\
Mucositis & $3(3.9)$ \\
Stomatitis & $1(1.3)$ \\
lleus & $1(1.3)$ \\
\hline
\end{tabular}

${ }^{*}$, there were no toxicities CTC grade 5. overall survival (either from the start of 1 st line or 2 nd line) were not significantly affected by a KRAS mutation. Other clinical parameters including age, gender, BMI, histology (adeno-carcinoma or NOS), as well as response to 1st line and length of ICI maintenance therapy were not associated with significantly different effects on efficacy parameters for $\mathrm{D}+\mathrm{R}$ treatment.

\section{Discussion}

The combination of chemotherapy plus ICI $(\mathrm{CT} x+\mathrm{ICI})$ has become the standard palliative 1st line treatment for driver negative metastatic NSCLC with more than four phase III trials demonstrating superiority of efficacy endpoints compared to chemotherapy alone $(9-11,13)$. However, progression remains the leading clinical problem and there is an urgent medical need for efficacious 2 nd line strategies to overcome resistance. As none of the pivotal trials had a predefined 2 nd line therapy, no prospective data are

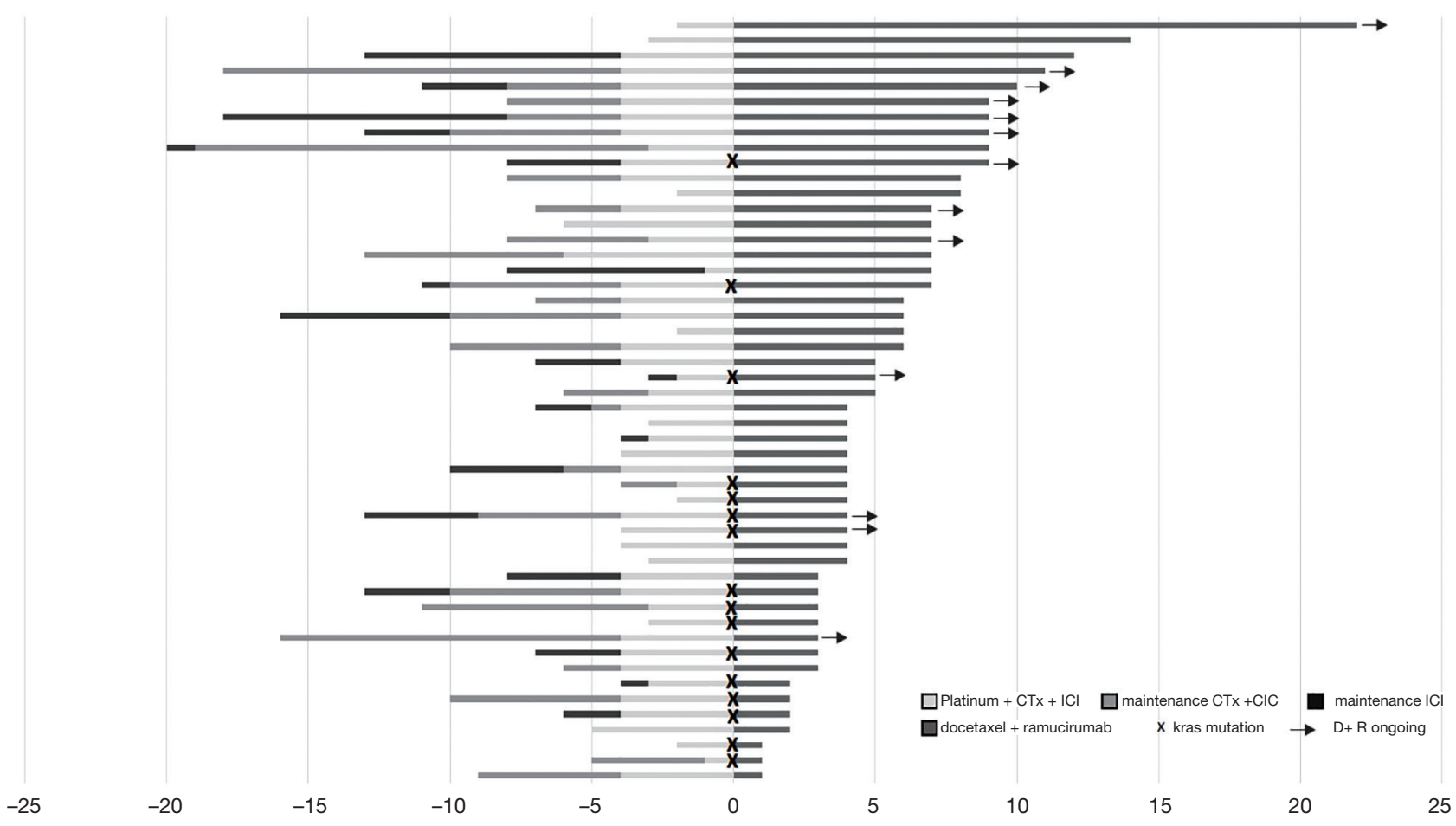

Figure 4 Treatment duration in cycles of therapy for 1 st and 2 nd line treatment in the subgroup of patients treated by platinum + pemetrexed + pembrolizumab 1st line $(\mathrm{N}=50)$. 1st line treatment left side is divided into platinum based CTx+ICI, maintenance therapy with $\mathrm{CTx}+\mathrm{ICI}$ and maintenance therapy with ICI monotherapy. D+R treatment is presented at the right side. Arrows define patients with $\mathrm{D}+\mathrm{R}$ ongoing, $\mathrm{X}$ defines patients with an underlying KRAS mutation. 
available for specific treatment sequences. Second line phase III trials evaluating docetaxel with an anti-angiogenetic substance, e.g., nintedanib $(\mathrm{D}+\mathrm{N})$ or ramucirumab $(\mathrm{D}+\mathrm{R})$ have each shown superiority to the comparator docetaxel monotherapy and led to approval of both combinations $(17,18)$. However, those trials were designed in the era before ICI were used in 1 st line treatment. For D+N a prospective German non-interventional trial (VARGADO) is underway and will provide insights to the efficacy of this sequence (19).

No prospective studies have been published to date for $\mathrm{D}+\mathrm{R}$ in this sequence. Thus, retrospective clinical data may provide at least some evidence for this therapeutic sequence. To the best of our knowledge, this cohort is the largest studied so far with a specific sequence of 1 st line CTX+ICI followed by $\mathrm{D}+\mathrm{R}$ in $2 \mathrm{nd}$ line. We showed that this sequence is feasible and effective in clinical routine. These results are consistent with the prospective data of the REVEL study on $\mathrm{D}+\mathrm{R}$ from the pre-ICI era and our efficacy and safety data are highly comparable with response rates being even higher (17). As long as no other trial data are available for 2 nd line therapy after $\mathrm{CT}+\mathrm{ICI}$, our data provide a solid basis for the use of $\mathrm{D}+\mathrm{R}$ in $2 \mathrm{nd}$ line after $\mathrm{CT} \mathrm{x}+\mathrm{ICI}$ in all histologies.

Nearly two-thirds of the patients in this study received platinum plus pemetrexed plus pembrolizumab in the 1st line setting. These patients represent a large, homogeneous and well-characterized subgroup, allowing for further indepth analyses. The data for clinical efficacy and safety were very favorable in this subgroup, although OS and PFS remained slightly inferior to the corresponding arm of the Keynote 189 trial, while response rates were comparable $(8,9)$. Patients in our analysis were treated in daily clinical practice and so were older and had more co-morbidities than those in the Keynote 189 trial. In addition, there may have been a negative selection bias in our patient population, since patients with exceptionally good response to 1 st line CTx+ICI may not have yet required 2nd line treatment. Furthermore, there were less tumors with a PDL1 TPS of $\geq 50 \%$ in our cohort than in the phase III trial with $6 \%$ versus $32 \%$, respectively.

We and others recently reported excellent PFS and OS data for $\mathrm{D}+\mathrm{R}$ given in $3 \mathrm{rd}$ line immediately after ICI monotherapy $(15,20-24)$. We have hypothesized that antiangiogenic agents not only act directly through VEGFmediated effects but also produce synergistic effects with chemotherapeutic agents and ICI agents for instance by contributing to better drug penetration through their action on tumor vessels $(15,25-27)$. Unfortunately, no clear evidence of such a synergistic effect could be observed in our analyses of the D+R second line cohort. Especially, no superior efficacy could be proven in patients with an objective response to 1st line therapy. However, there was at least a trend toward longer PFS with $\mathrm{D}+\mathrm{R}$ in patients with moderate (1-49\%) and high (>50\%) PD-L1 TPS scores versus tumors negative for PD-L1. Knowing that ICI bind to the PD-1 receptor up to two months after the last infusion of the drug, this could be a hint for the synergistic effect described above at least in PD-L1 high expressing tumors (28).

Most patients in the platinum plus pemetrexed plus pembrolizumab sub-cohort had available KRAS mutation data. In line with results from the KEYNOTE-189 trial, KRAS status had no impact on 1st-line therapy (29). However, a positive KRAS status was significantly associated with worse median PFS in 2 nd-line $D+R$ and was also an independent predictor of worse PFS in multivariate Cox regression analysis. To date, there is little evidence on the role of KRAS status in D+R therapy (30). Although KRAS mutations might increase the expression of VEGF, VEGFR1 and VEGFR2 in various tumors including NSCLC, the underlying mechanisms is still unclear (31-33). In vitro data of ramucirumab showed inferior tumor regression in a KRAS mutated xenograft tumor (NCI-H2122) which could be significantly increased by the combination of ramucirumab with a panRAF inhibitor (34). In addition, a KRAS mutation was a significantly negative predictor for response and time on treatment when bevacizumab, a VEGF antibody, was given in combination with carboplatin and docetaxel in NSCLC (35). These observations support our findings. Nevertheless, the significance of KRAS status in this situation has not yet been conclusively determined, and KRAS status should be analyzed systematically in future studies investigating antiangiogenic combination therapies after Ctx+ICI (36).

Some potential limitations of our study must be addressed. This was a retrospective study and some underreporting of potential side effects may have occurred. Since severe side effects are usually well documented during clinical routine, underreporting may have mainly affected the documentation of those side effects not immediately apparent in routine care. Furthermore, there may have been some negative selection bias since patients with limited effect of 1 st line treatment may have been over-represented in our cohort. Accordingly, there remains some uncertainty whether $\mathrm{D}+\mathrm{R}$ is an effective 2 nd line therapy for patients 
with a particularly good response to $\mathrm{CTx}+\mathrm{ICI} 1$ st line therapy. In this study, patient response to treatment was assessed by criteria used during routine care at participating centers and was not collected in a predefined, standardized manner by independent investigators. Therefore, some variability between centers may be present for PFS, but this would not have affected survival data. KRAS mutation status was not reported for about one third of the patients. However, of those $20.4 \%$ presented with a squamous cell carcinoma. We do not have evidence that there was a systematic error in this regard, so the patients with unknown KRAS status should be a representative sample from the overall cohort.

Overall, our results clearly demonstrate a superb OS and PFS from 2nd line D+R therapy after 1st-line therapy with CTx+ICI for metastatic NSCLC of all histologies. This effect was even slightly more pronounced in a large subgroup treated with platinum plus pemetrexed plus pembrolizumab in 1st line. Some early evidence was found that patients with a KRAS mutation may have significantly less response to $\mathrm{D}+\mathrm{R}$ after $\mathrm{CT} \mathrm{x}+\mathrm{ICI}$. Further systematic studies are needed to identify robust criteria for patient selection for 2 nd line therapy with $\mathrm{D}+\mathrm{R}$ after $\mathrm{CT}$ s+ICI.

\section{Acknowledgments}

We thank all the patients who participated in this study. Funding: None.

\section{Footnote}

Reporting Checklist: The authors have completed the STROBE reporting checklist. Available at https://dx.doi. org/10.21037/tlcr-21-197

Data Sharing Statement: Available at https://dx.doi. org/10.21037/tlcr-21-197

Peer Review File: Available at https://dx.doi.org/10.21037/ tlcr-21-197

Conflicts of Interest: All authors have completed the ICMJE uniform disclosure form (available at https://dx.doi. org/10.21037/tlcr-21-197). Albrecht Stenzinger serves as an unpaid editorial board member of Translational Lung Cancer Research from Sep 2019 to Sep 2021. The following authors received honoraria for lectures, presentation, speakers bureaus, manuscript writing or educational events:
WMB from AstraZeneca, Boehringer, Novartis, MSD, BMS, Roche and Lilly; MR from Amgen, AstraZeneca, BMS, Boehringer, Lilly, Minde, Merck, MSD, Novartis, Pfizer and Roche; AR from AbbVIe, AstraZeneca, BMS, Boehringer, Lilly, MSD, Novartis, Pfizer and Roche; CW from Boehringer, Lilly, MSD, Roche, AstraZeneca, BMS, Sanofi Aventis and Takeda; GHW from Boehringer, Lilly, MSD, Roche, AstraZeneca, BMS, Sanofi Aventis, Takeda, Glaxo and Berlin Chemie; PC from Roche, Takeda, AstraZeneca and Novartis; AS from Aignostics, Bayer, Thermo Fisher, Illumina, AstraZeneca, Novartis, Pfizer, Roche, Seattle Genetics, MSD, BMS, Takeda and Lilly; AT from GSK, Novartis, Amgen, BMS, MSD, Lilly, Pfizer, Boehringer, Roche, Takeda and Celgene; JHF from AstraZeneca, Boehringer, Novartis, MSD, BMS and Roche. The following authors received support for attending meetings and/or travel: WMB from Boehringer, AstraZeneca and Roche; CW from Boehringer, MSD, Roche, AstraZeneca and BMS; GW from Boehringer, MSD, Roche, AstraZeneca and Berlin Chemie; PC from AstraZeneca, Takeda, Novartis and Lilly; AT from GSK, Novartis, Amgen, MSD, BMS, Lilly, Pfizer, Boehringer, Roche, Takeda and Celgene; JHF from Boehringer and AstraZeneca. The following authors participated on a data safety monitoring board or an advisory board: WMB on AstraZeneca, Boehringer, Novartis, MSD, Lilly, BMS and Roche; JK on Roche, Boehringer, BMS, Merck, Amgen, Takeda and Lilly (all outside the submitted work and all without receiving any personal fees; $\mathrm{CW}$ on Boehringer, MSD, Roche, AstraZeneca and MBS; GHW on Boehringer, MSD, Roche, AstraZeneca and BMS; PC on Pfizer, Chugai, Boehringer and Roche; AT on GSK, Novartis, Amgen, MSD, BMS, Lilly, Pfizer, Boehringer, Roche, Takeda, Celgene and AIO Leadership Group; PH on Lilly, MSD, BMS, AstraZeneca, Takeda, Roche, Boehringer and Pfizer; JHF on AstraZenca. The following authors receipt equipment, materials, drugs, medical writing, gifts or other services: WMB from Boehringer (for medical writing); JHF from Novartis (for medical writing); the following authors received consulting fees: AR from AbbVie, AstraZeneca, BMS, Boehringer, Lilly, MSD, Novartis, Pfizer and Roche. The following authors received grants or contracts form any entity: PC from Roche, Takeda, AstraZeneca and Novartis (all research grants to the institution; AT from AstraZeneca (research grant). The other authors have no conflicts of interest to declare.

Ethical Statement: The authors are accountable for all aspects 
of the work ensuing that questions related to the accuracy or integrity of any part of the work are appropriately investigated and resolved. This study was conducted in accordance with the Declaration of Helsinki (as revised in 2013). This study was approved by the Institutional Review Board of Paracelsus Medical University Nuremberg (IRB2019-014). Individual consent for this retrospective analysis was waived.

Open Access Statement: This is an Open Access article distributed in accordance with the Creative Commons Attribution-NonCommercial-NoDerivs 4.0 International License (CC BY-NC-ND 4.0), which permits the noncommercial replication and distribution of the article with the strict proviso that no changes or edits are made and the original work is properly cited (including links to both the formal publication through the relevant DOI and the license). See: https://creativecommons.org/licenses/by-nc-nd/4.0/.

\section{References}

1. Borghaei H, Paz-Ares L, Horn L, et al. Nivolumab versus Docetaxel in Advanced Nonsquamous Non-Small-Cell Lung Cancer. N Engl J Med 2015;373:1627-39.

2. Fehrenbacher L, Spira A, Ballinger M, et al. Atezolizumab versus docetaxel for patients with previously treated non-small-cell lung cancer (POPLAR): a multicentre, open-label, phase 2 randomised controlled trial. Lancet 2016;387:1837-46.

3. Herbst RS, Baas P, Kim DW, et al. Pembrolizumab versus docetaxel for previously treated, PD-L1-positive, advanced non-small-cell lung cancer (KEYNOTE-010): a randomised controlled trial. Lancet 2016;387:1540-50.

4. Horn L, Spigel DR, Vokes EE, et al. Nivolumab Versus Docetaxel in Previously Treated Patients With Advanced Non-Small-Cell Lung Cancer: Two-Year Outcomes From Two Randomized, Open-Label, Phase III Trials (CheckMate 017 and CheckMate 057). J Clin Oncol 2017;35:3924-33.

5. Reck M, Rodriguez-Abreu D, Robinson AG, et al. Pembrolizumab versus Chemotherapy for PD-L1Positive Non-Small-Cell Lung Cancer. N Engl J Med 2016;375:1823-33.

6. Rittmeyer A, Barlesi F, Waterkamp D, et al. Atezolizumab versus docetaxel in patients with previously treated non-small-cell lung cancer (OAK): a phase 3, openlabel, multicentre randomised controlled trial. Lancet 2017;389:255-65.
7. Gettinger S, Horn L, Jackman D, et al. Five-Year FollowUp of Nivolumab in Previously Treated Advanced NonSmall-Cell Lung Cancer: Results From the CA209-003 Study. J Clin Oncol 2018;36:1675-84.

8. Gadgeel S, Rodriguez-Abreu D, Speranza G, et al. Updated Analysis From KEYNOTE-189: Pembrolizumab or Placebo Plus Pemetrexed and Platinum for Previously Untreated Metastatic Nonsquamous Non-Small-Cell Lung Cancer. J Clin Oncol 2020;38:1505-17.

9. Gandhi L, Rodriguez-Abreu D, Gadgeel S, et al. Pembrolizumab plus Chemotherapy in Metastatic NonSmall-Cell Lung Cancer. N Engl J Med 2018;378:2078-92.

10. Paz-Ares L, Luft A, Vicente D, et al. Pembrolizumab plus Chemotherapy for Squamous Non-Small-Cell Lung Cancer. N Engl J Med 2018;379:2040-51.

11. Reck M, Mok TSK, Nishio M, et al. Atezolizumab plus bevacizumab and chemotherapy in non-small-cell lung cancer (IMpower150): key subgroup analyses of patients with EGFR mutations or baseline liver metastases in a randomised, open-label phase 3 trial. Lancet Respir Med 2019;7:387-401.

12. Socinski MA, Jotte RM, Cappuzzo F, et al. Atezolizumab for First-Line Treatment of Metastatic Nonsquamous NSCLC. N Engl J Med 2018;378:2288-301.

13. Paz-Ares L, Ciuleanu TE, Cobo M, et al. First-line nivolumab plus ipilimumab combined with two cycles of chemotherapy in patients with non-small-cell lung cancer (CheckMate 9LA): an international, randomised, openlabel, phase 3 trial. Lancet Oncol 2021;22:198-211.

14. Santos ES. Treatment options after first-line immunotherapy in metastatic NSCLC. Expert Rev Anticancer Ther 2020;20:221-8.

15. Brueckl WM, Reck M, Rittmeyer A, et al. Efficacy of Docetaxel Plus Ramucirumab as Palliative Third-Line Therapy Following Second-Line Immune-CheckpointInhibitor Treatment in Patients With Non-SmallCell Lung Cancer Stage IV. Clin Med Insights Oncol 2020;14:1179554920951358.

16. Eisenhauer EA, Therasse P, Bogaerts J, et al. New response evaluation criteria in solid tumours: revised RECIST guideline (version 1.1). Eur J Cancer 2009;45:228-47.

17. Garon EB, Ciuleanu TE, Arrieta O, et al. Ramucirumab plus docetaxel versus placebo plus docetaxel for secondline treatment of stage IV non-small-cell lung cancer after disease progression on platinum-based therapy (REVEL): a multicentre, double-blind, randomised phase 3 trial. Lancet 2014;384:665-73.

18. Reck M, Kaiser R, Mellemgaard A, et al. Docetaxel plus 
nintedanib versus docetaxel plus placebo in patients with previously treated non-small-cell lung cancer (LUMELung 1): a phase 3, double-blind, randomised controlled trial. Lancet Oncol 2014;15:143-55.

19. Grohe C, Gleiber W, Krüger S, et al. Efficacy and safety of nintedanib plus docetaxel in lung adenocarcinoma patients following treatment with immune checkpoint inhibitors: updated results of the ongoing NIS VARGADO (NCT02392455). Ann Oncol 2019;30:mdz449.020.

20. Harada D, Takata K, Mori S, et al. Previous Immune Checkpoint Inhibitor Treatment to Increase the Efficacy of Docetaxel and Ramucirumab Combination Chemotherapy. Anticancer Res 2019;39:4987-93.

21. Kato R, Hayashi H, Chiba Y, et al. Propensity scoreweighted analysis of chemotherapy after PD-1 inhibitors versus chemotherapy alone in patients with non-small cell lung cancer (WJOG10217L). J Immunother Cancer 2020;8:e000350.

22. Shiono A, Kaira K, Mouri A, et al. Improved efficacy of ramucirumab plus docetaxel after nivolumab failure in previously treated non-small cell lung cancer patients. Thorac Cancer 2019;10:775-81.

23. Tozuka T, Kitazono S, Sakamoto H, et al. Addition of ramucirumab enhances docetaxel efficacy in patients who had received anti-PD-1/PD-L1 treatment. Lung Cancer 2020;144:71-5.

24. Yoshimura A, Yamada T, Okuma Y, et al. Retrospective analysis of docetaxel in combination with ramucirumab for previously treated non-small cell lung cancer patients. Transl Lung Cancer Res 2019;8:450-60.

25. Fukumura D, Jain RK. Tumor microvasculature and microenvironment: targets for anti-angiogenesis and normalization. Microvasc Res 2007;74:72-84.

26. Liang H, Wang M. Prospect of immunotherapy combined with anti-angiogenic agents in patients with advanced non-small cell lung cancer. Cancer Manag Res 2019;11:7707-19.

27. Tong RT, Boucher Y, Kozin SV, et al. Vascular normalization by vascular endothelial growth factor receptor 2 blockade induces a pressure gradient across the vasculature and improves drug penetration in tumors. Cancer Res 2004;64:3731-6.

28. Brahmer JR, Drake CG, Wollner I, et al. Phase I study of single-agent anti-programmed death-1 (MDX1106) in refractory solid tumors: safety, clinical activity, pharmacodynamics, and immunologic correlates. J Clin
Oncol 2010;28:3167-75.

29. Gadgeel S, Rodrigues-Abreu D, Felip E, et al. KRAS mutational status and efficacy in KEYNOTE-189: pembrolizumab (pembro) plus chemotherapy (chemo) vs placebo plus chemo as first-line therapy for metastatic non-squamous NSCLC. Ann Oncol 2019;30:xi64.

30. Brueckl WM, Ficker JH, Zeitler G. Clinically relevant prognostic and predictive markers for immune-checkpointinhibitor (ICI) therapy in non-small cell lung cancer (NSCLC). BMC Cancer 2020;20:1185.

31. Mancikova V, Inglada-Perez L, Curras-Freixes $M$, et al. VEGF, VEGFR3, and PDGFRB protein expression is influenced by RAS mutations in medullary thyroid carcinoma. Thyroid 2014;24:1251-5.

32. Schimanski CC, Zimmermann T, Schmidtmann I, et al. K-ras mutation status correlates with the expression of VEGFR1, VEGFR2, and PDGFRalpha in colorectal cancer. Int J Colorectal Dis 2010;25:181-6.

33. Yuan XH, Yang J, Wang XY, et al. Association between EGFR/KRAS mutation and expression of VEGFA, VEGFR and VEGFR2 in lung adenocarcinoma. Oncol Lett 2018;16:2105-12.

34. Wu W, Stewart J, King C, et al. Combined inhibition of pan-RAF and VEGFR-2 mediates antitumor activity in KRAS mutant NSCLC through enhanced inhibition of tumor angiogenesis and growth. Am Assoc Cancer Res (AACR) 2106;76:76.

35. Domine M, Rojo F, Izarzugaza Y, et al. Kras status as predictive marker of response and time to progression in EGFR wild-type stage IV NSCLC patients treated with platin-docetaxel-bevacizumab. Ann Oncol 2021;23:134.

36. Chen H, Zhao J. KRAS oncogene may be another target conquered in non-small cell lung cancer (NSCLC). Thorac Cancer 2020;11:3425-35.

Cite this article as: Brueckl WM, Reck M, Rittmeyer A, Kollmeier J, Wesseler C, Wiest GH, Christopoulos P, Stenzinger A, Tufman A, Hoffknecht P, Ulm B, Reich F, Ficker JH, Laack E. Efficacy of docetaxel plus ramucirumab as palliative second-line therapy following first-line chemotherapy plus immune-checkpoint-inhibitor combination treatment in patients with non-small cell lung cancer (NSCLC) UICC stage IV. Transl Lung Cancer Res 2021;10(7):3093-3105. doi: $10.21037 /$ tlcr-21-197 

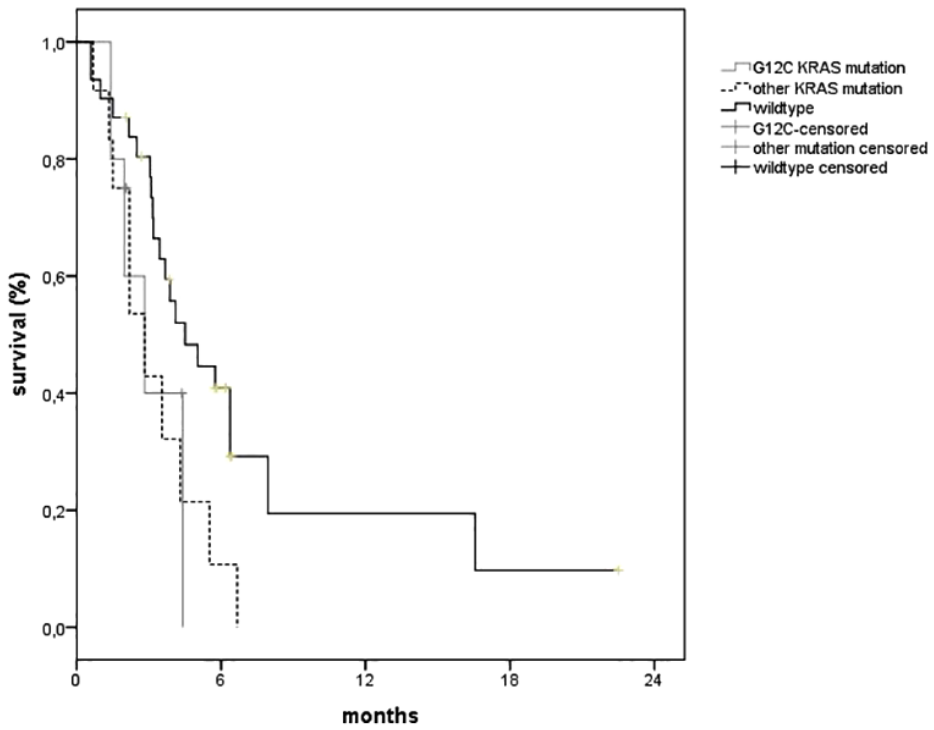

Figure S1 Kaplan-Meier curve for PFS due to KRAS status (wild-type versus G12C versus other KRAS mutations). 